\title{
COMPLEX HERMITE POLYNOMIALS: THEIR COMBINATORICS AND INTEGRAL OPERATORS
}

\author{
MOURAD E. H. ISMAIL AND PLAMEN SIMEONOV \\ (Communicated by Jim Haglund)
}

\begin{abstract}
We consider two types of Hermite polynomials of a complex variable. For each type we obtain combinatorial interpretations for the linearization coefficients of products of these polynomials. We use the combinatorial interpretations to give new proofs of several orthogonality relations satisfied by these polynomials with respect to positive exponential weights in the complex plane. We also construct four integral operators of which the first type of complex Hermite polynomials are eigenfunctions and we identify the corresponding eigenvalues. We prove that the products of these complex Hermite polynomials are complete in certain $L_{2}$-spaces.
\end{abstract}

\section{INTRODUCTION}

We consider two types of complex Hermite polynomials. The first type is simply the Hermite polynomials in the complex variable $z$, that is, $\left\{H_{n}(z)\right\}$. These polynomials have been introduced in the study of coherent states [4], 6]. They are defined by [18, [11,

$$
H_{n}(z)=\sum_{k=0}^{\lfloor n / 2\rfloor} \frac{n !(-1)^{k}}{k !(n-2 k) !}(2 z)^{n-2 k}, \quad z=x+i y .
$$

The second type are the polynomials $\left\{H_{m, n}(z, \bar{z})\right\}$ defined by the generating function

$$
\sum_{m, n=0}^{\infty} H_{m, n}(z, \bar{z}) \frac{u^{m} v^{n}}{m ! n !}=\exp (u z+v \bar{z}-u v) .
$$

These polynomials were introduced by Itô [14] and also appear in [7, 1], 4, 19], and [8. They are essentially the same as in [5, (2.6.6)]. Equation (1.2) yields the

Received by the editors January 25, 2013 and, in revised form, May 31, 2013 and July 18, 2013.

2010 Mathematics Subject Classification. Primary 05A15, 05A18, 33C45, 45P05; Secondary $42 \mathrm{~A} 65$.

Key words and phrases. Complex Hermite polynomials, matchings of multisets, orthogonality, combinatorics of linearization of products, eigenvalues, eigenfunctions, integral operators, completeness.

The first author's research was supported by the NPST Program of King Saud University; project number 10-MAT1293-02 and King Saud University in Riyadh and by the Research Grants Council of Hong Kong grant \# CityU 1014111. 
explicit formula

$$
H_{m, n}(z, \bar{z})=\sum_{k=0}^{m \wedge n}(-1)^{k} k !\left(\begin{array}{l}
m \\
k
\end{array}\right)\left(\begin{array}{l}
n \\
k
\end{array}\right) z^{m-k} \bar{z}^{n-k} .
$$

One simple combinatorial interpretation of the polynomials $\left\{H_{n}(x / 2)\right\}$ is apparent from (1.1):

$$
H_{n}(x / 2)=\sum_{\pi \in \mathcal{M}(n)}(-1 / 2)^{\operatorname{edge}(\pi)} x^{\mathrm{fix}(\pi)}
$$

where $\mathcal{M}(n)$ is the set of all unordered matchings $\pi$ of $n$ vertices, edge $(\pi)$ is the number of edges in $\pi$ (pairs of matched by $\pi$ vertices) and fix $(\pi)$ is the number of vertices fixed by $\pi$ (vertices not matched to other vertices by $\pi$ ).

It is clear that the explicit formula (1.1) is equivalent to the generating function ([18, [1]),

$$
\sum_{n=0}^{\infty} \frac{H_{n}(z)}{n !} t^{n}=\exp \left(2 z t-t^{2}\right) .
$$

The complex Hermite polynomials $\left\{H_{n}(z)\right\}$ satisfy the orthogonality relation ([20], [15]),

$$
\int_{\mathbb{R}^{2}} H_{m}(x+i y) H_{n}(x-i y) e^{-a x^{2}-b y^{2}} d x d y=\frac{\pi}{\sqrt{a b}} 2^{n} n !\left(\frac{a+b}{a b}\right)^{n} \delta_{m, n}
$$

where

$$
0<a<b, \quad \frac{1}{a}=1+\frac{1}{b} .
$$

The polynomials $\left\{H_{m, n}(z, \bar{z})\right\}$ satisfy the orthogonality relation ([8]),

$$
\frac{1}{\pi} \int_{\mathbb{R}^{2}} H_{m, n}(x+i y, x-i y) \overline{H_{p, q}(x+i y, x-i y)} e^{-x^{2}-y^{2}} d x d y=m ! n ! \delta_{m, p} \delta_{n, q} .
$$

Let $k$ be a fixed positive integer and let $\mathbf{n}=\left(n_{1}, \ldots, n_{k}\right)$ be a $k$-tuple of nonnegative integers, that is, $\mathbf{n} \in \mathbb{N}_{0}^{k}$. Set $|\mathbf{n}|=\sum_{j=1}^{k} n_{j}$. Azor, Gillis, and Victor [3], and Godsil [9], independently, found a combinatorial interpretation of the integrals

$$
A(\mathbf{n})=\frac{1}{\sqrt{\pi}} 2^{-\frac{1}{2}|\mathbf{n}|} \int_{\mathbb{R}} e^{-x^{2}} \prod_{j=1}^{k} H_{n_{j}}(x) d x
$$

as the number of inhomogeneous perfect matchings of a multiset with $k$ sets (components) of sizes (number of elements) $n_{1}, \ldots, n_{k}$.

By multiset we mean a collection of sets or components. The elements of each set are labeled (distinguishable). A matching of one or more multisets is perfect if each element is matched to another element, and inhomogeneous if each element is matched to an element from a different set. The pairs of matched elements (edges) are unordered (unoriented). The matching is weighted if a weight is assigned to each pair of matched elements in the matching, and the weight of the matching is the product of the weights of its edges. The total weight of a collection of matchings is the sum of their weights. In what follows we shall often skip the adjective "inhomogeneous" when it is clear from the context that the matchings considered are inhomogeneous. We say that a multiset has size $\mathbf{n}=\left(n_{1}, \ldots, n_{k}\right) \in \mathbb{N}_{0}^{k}$ if it 
has $k$ components of respective sizes $n_{1}, \ldots, n_{k}$. We allow some but not all of the multiset components to be empty.

The numbers in (1.9) are also the coefficients in the linearization of products of Hermite polynomials when expanded in Hermite polynomials; see [2] and 11. Chapter 9] for references and motivation. Large parameter asymptotics of such coefficients were studied in 13 from a combinatorial point of view. The Hermite polynomials of a real variable have combinatorial properties. The combinatorics of their moments, explicit representation, and three term recurrence relation is available in Viennot's Lecture Notes 21. The combinatorics of the general case of Sheffer orthogonal polynomials is in [16]; see also [22. A general approach to the combinatorics of linearization coefficients is in [16].

The first combinatorial interpretation of complex Hermite polynomials that we will discuss involves two colored multisets. The following theorem will be proved in Section 2.

Theorem 1.1. Suppose that we have two colored multisets. The first, of color I, has size $\mathbf{m} \in \mathbb{N}_{0}^{k}$ and the second, of color II, has size $\mathbf{n} \in \mathbb{N}_{0}^{k}$. We match elements from different sets, and to each pair of matched elements we assign weight 1 if the elements have the same color. We assign weight $1 / a+1 / b$ if the elements have different colors.

Then the total weight of all perfect matchings of this type is the number $B(\mathbf{m}, \mathbf{n})$ defined by

$$
B(\mathbf{m}, \mathbf{n})=\frac{\sqrt{a b}}{\pi} 2^{-\frac{1}{2}(|\mathbf{m}|+|\mathbf{n}|)} \int_{\mathbb{R}^{2}} \prod_{j=1}^{k}\left[H_{m_{j}}(x+i y) H_{n_{j}}(x-i y)\right] e^{-a x^{2}-b y^{2}} d x d y .
$$

Observe that the orthogonality relation (1.6) follows immediately from Theorem 1.1 .

The following theorem was first proved by K. Gorska [10].

Theorem 1.2. Let $a$ and $b$ satisfy conditions (1.7). Then the complex Hermite polynomials $\left\{H_{n}(z)\right\}$ satisfy the orthogonality relations

$$
\begin{gathered}
\int_{\mathbb{R}^{2}} H_{m}(x+i y) H_{n}(x+i y) e^{-a x^{2}-b y^{2}} d x d y \\
=\int_{\mathbb{R}^{2}} H_{m}(x-i y) H_{n}(x-i y) e^{-a x^{2}-b y^{2}} d x d y=\frac{\pi}{\sqrt{a b}} 2^{n} n ! \delta_{m, n} .
\end{gathered}
$$

The orthogonality relations in Theorem 1.2 play a fundamental role in the construction of coherent states (for example see [20]).

A generating function for the numbers $\{B(\mathbf{m}, \mathbf{n})\}$ is derived in Section 2. Using this generating function, we establish the combinatorial interpretation of these numbers given by Theorem 1.1 and then we show that the orthogonality relations (1.6) and (1.11) are simple consequences of this combinatorial interpretation and the generating function (2.1). In Section 2 we also give a generalization of the two-color multiset combinatorics to arbitrary number of colored multisets. At the end of Section 2 we treat a special case of this multiset combinatorics when each multiset consists of a single set.

Concerning the polynomials $\left\{H_{m, n}(z, \bar{z})\right\}$, the following combinatorial result is proved in Section 3. 
Theorem 1.3. Suppose that we have two color I multisets $B_{1}$ and $B_{2}$ and two color II multisets $R_{1}$ and $R_{2}$, each having $k$ components. Multisets $B_{1}$ and $B_{2}$ have sizes $\mathbf{m}$ and $\mathbf{p} \in \mathbb{N}_{0}^{k}$ and multisets $R_{1}$ and $R_{2}$ have sizes $\mathbf{n}$ and $\mathbf{q} \in \mathbb{N}_{0}^{k}$, respectively. Consider all inhomogeneous perfect matching of these four multisets where each element is matched to an element of different color. Elements from the same index components of $B_{1}$ and $R_{1}$ cannot match each other, and the same restriction holds for $B_{2}$ and $R_{2}$. Then the number of perfect matchings of this type is

$I(\mathbf{m}, \mathbf{n}, \mathbf{p}, \mathbf{q})=\frac{1}{\pi} \int_{\mathbb{R}^{2}} \prod_{j=1}^{k} H_{m_{j}, n_{j}}(x+i y, x-i y) H_{p_{j}, q_{j}}(x+i y, x-i y) e^{-x^{2}-y^{2}} d x d y$.

In Section 3 we first derive an exponential generating function for integrals involving products of polynomials $H_{m, n}(z, \bar{z})$, and as a particular case, a generating function for the numbers $\{I(\mathbf{m}, \mathbf{n}, \mathbf{p}, \mathbf{q})\}$. The former generating function is used to prove a more general version of Theorem 1.3 for even number of colored multisets which is then specialized to the case of four colored multisets in Theorem 1.3.

In Section 4 we construct two integral operators and two operators of Fourier type acting on weighted $L_{2}$-spaces with weights $e^{-a x^{2}-b y^{2}}$ and constants $a$ and $b$ satisfying (1.7). We show that the polynomials $\left\{H_{n}(x+i y)\right\}$ are eigenfunctions for these operators and we find the corresponding eigenvalues. In Section 4 we also prove that the set $\left\{H_{m}(x+i y) H_{n}(x-i y)\right\}$ is complete in $L_{2}\left(\mathbb{R}^{2}, e^{-a x^{2}-b y^{2}}\right)$, and we compute the moments of $e^{-a x^{2}-b y^{2}}$.

In our proofs we shall use the following version of the exponential formula for exponential generating functions [17.

Theorem 1.4. Let $S_{1}, \ldots, S_{k}$ be sets of sizes $\left|S_{j}\right|=n_{j}, j=1, \ldots, k$, and let $\mathcal{M}(\mathbf{n})$ be the collection of all inhomogeneous perfect matchings of the elements of these $k$ sets, where $\mathbf{n}=\left(n_{1}, \ldots, n_{k}\right)$. Let also $\{w\}=\left\{w_{j_{1}, j_{2}}, 1 \leq j_{1}<j_{2} \leq k\right\} \subset \mathbb{C}$. To each pair of matched objects (edge) from sets $S_{j_{1}}$ and $S_{j_{2}}\left(\right.$ with $1 \leq j_{1}<j_{2} \leq k$ ) the weight $w_{j_{1}, j_{2}}$ is assigned, and to each $\pi \in \mathcal{M}(\mathbf{n})$ the weight that is the product of the weights of the edges in $\pi$ is assigned. Let $A_{\{w\}}(\mathbf{n})$ be the sum of the weights of all perfect matchings in $\mathcal{M}(\mathbf{n})$. Then

$$
\sum_{n_{1}, \ldots, n_{k}=0}^{\infty} A_{\{w\}}(\mathbf{n}) \prod_{j=1}^{k} \frac{x_{j}^{n_{j}}}{n_{j} !}=\exp \left[\sum_{1 \leq j_{1}<j_{2} \leq k} w_{j_{1}, j_{2}} x_{j_{1}} x_{j_{2}}\right] .
$$

\section{Combinatorial interpretations of the polynomials $\left\{H_{n}(z)\right\}$}

We first derive an exponential generating function of the numbers $\{B(\mathbf{m}, \mathbf{n})\}$.

Theorem 2.1. Suppose that $a$ and $b$ satisfy (1.7). Then

$$
\begin{gathered}
\sum_{m_{1}, \ldots, m_{k}, n_{1}, \ldots, n_{k}=0}^{\infty} B(\mathbf{m}, \mathbf{n}) \prod_{j=1}^{k} \frac{s_{j}^{m_{j}} t_{j}^{n_{j}}}{m_{j} ! n_{j} !} \\
=\exp \left[\sum_{1 \leq i<j \leq k}\left(s_{i} s_{j}+t_{i} t_{j}\right)+\frac{a+b}{a b} \sum_{1 \leq i, j \leq k} s_{i} t_{j}\right] .
\end{gathered}
$$


Proof. Using (1.5) and (1.10) we write the left hand side of (2.1) as

$$
\begin{aligned}
& \frac{\sqrt{a b}}{\pi} \int_{\mathbb{R}^{2}} e^{-a x^{2}-b y^{2}} \prod_{j=1}^{k} \exp \left[-s_{j}^{2} / 2+\sqrt{2}(x+i y) s_{j}-t_{j}^{2} / 2+\sqrt{2}(x-i y) t_{j}\right] d x d y \\
& =\frac{\sqrt{a b}}{\pi} \exp \left[-\frac{1}{2} \sum_{j=1}^{k}\left(s_{j}^{2}+t_{j}^{2}\right)+\frac{1}{2 a}\left[\sum_{j=1}^{k}\left(s_{j}+t_{j}\right)\right]^{2}-\frac{1}{2 b}\left[\sum_{j=1}^{k}\left(s_{j}-t_{j}\right)\right]^{2}\right] \\
& \quad \times \int_{\mathbb{R}^{2}} \exp \left[-a\left[x-\frac{1}{a \sqrt{2}} \sum_{j=1}^{k}\left(s_{j}+t_{j}\right)\right]^{2}-b\left[y-\frac{i}{b \sqrt{2}} \sum_{j=1}^{k}\left(s_{j}-t_{j}\right)\right]^{2}\right] d x d y .
\end{aligned}
$$

Notice that by (1.7) the exponential expression in the second line of (2.2) reduces to the right hand side of (2.1). Next, for $\alpha>0$ and $\beta \in \mathbb{C}$ we have the integral evaluation

$$
\int_{\mathbb{R}} e^{-\alpha(y-\beta)^{2}} d y=\sqrt{\pi / \alpha}
$$

If $\beta \in \mathbb{R}$, (2.3) follows by setting $x=\sqrt{2 \alpha}(y-\beta)$ and using the Gaussian integral $\frac{1}{\sqrt{2 \pi}} \int_{\mathbb{R}} e^{-x^{2} / 2} d x=1$. If $\beta \notin \mathbb{R},(2.3)$ follows by taking the limit $M \rightarrow \infty$ in

$$
\int_{\gamma(M)} e^{-\alpha z^{2}} d z=0
$$

where $M>0$ and $\gamma(M)$ is the simple, closed, positively oriented contour formed by the boundary of the parallelogram with vertices $\pm M$ and $\pm M-\beta$.

By (2.3), the last integral in (2.2) equals $\pi / \sqrt{a b}$. This completes the verification of (2.1).

Proof of Theorem 1.1. The theorem follows from the generating function (2.1) and Theorem 1.4.

Orthogonality relation (1.6) follows from the special case $k=1$ of Theorem 1.1 and Theorem 2.1.

Proof of Theorem 1.2. Orthogonality relations (1.11) follow from Theorems 1.1 and 2.1 with $k=2$ by taking either $\mathbf{m}=(m, n)$ and $\mathbf{n}=(0,0)$ or $\mathbf{m}=(0,0)$ and $\mathbf{n}=(m, n)$.

We now come to the combinatorics of the polynomials $\left\{H_{n}(x+y)\right\}$.

Theorem 2.2. The Hermite polynomials $\left\{H_{n}(x+y)\right\}$ have the explicit form

$$
\begin{aligned}
& H_{n}((x+y) / 2)=\sum_{\pi \in \mathcal{M}(n)}(-1 / 2)^{\operatorname{edge}(\pi)}(x+y)^{\mathrm{fix}(\pi)} \\
= & \sum_{\pi \in \mathcal{M}(n)}(-1 / 2)^{\operatorname{edge}(\pi)}\left(\begin{array}{c}
\operatorname{fix}(\pi) \\
\operatorname{fixRed}(\pi)
\end{array}\right) x^{\operatorname{fixRed}(\pi)} y^{\operatorname{fixBlue}(\pi)}
\end{aligned}
$$

where fixRed $(\pi)$ is the number of fixed points which we color red and fixBlue $(\pi)$ is the number of fixed points which we color blue.

Proof. Equation (2.4) follows from equation (1.4) in which we arbitrarily color the fixed points of $\pi$ in red or blue colors. 
2.1. Combinatorics involving colored multisets. The generating function in Theorem 2.1 and the combinatorial interpretation in Theorem 1.1 can be extended to any number of multisets. As a multivariable analog of the numbers in (1.10) we define the numbers

$$
\begin{aligned}
& B\left(\mathbf{n}_{1}, \ldots, \mathbf{n}_{s}\right) \\
& \quad=\frac{2^{-\alpha_{s} \sum_{\nu=1}^{s}\left|\mathbf{n}_{\nu}\right|} \prod_{l=1}^{s} a_{l}^{1 / 2}}{\pi^{s / 2}} \int_{\mathbb{R}^{s}} \prod_{\nu=1}^{s} \prod_{j=1}^{k} H_{n_{\nu, j}}\left(L_{\nu}(\vec{x})\right) e^{-\sum_{l=1}^{s} a_{l} x_{l}^{2}} d x_{1} \cdots d x_{s}
\end{aligned}
$$

where $\mathbf{n}_{\nu}=\left(n_{\nu, 1}, \ldots, n_{\nu, k}\right) \in \mathbb{N}_{0}^{k}, \nu=1, \ldots, s, \alpha_{s} \geq 0, a_{l}>0, l=1, \ldots, s$, $\vec{x}=\left(x_{1}, \ldots, x_{s}\right)$, and

$$
L_{\nu}(\vec{x})=\sum_{l=1}^{s} \omega_{\nu, l} x_{l}, \quad \nu=1, \ldots, s
$$

are linear functions with complex coefficients satisfying the conditions

$$
\sum_{l=1}^{s} \frac{\omega_{\nu, l}^{2}}{a_{l}}=1, \quad \nu=1, \ldots, s .
$$

Theorem 2.3. The numbers defined by (2.5) -(2.7) have the generating function

$$
\begin{gathered}
\sum_{\substack{n_{\nu, j}=0 \\
1 \leq \nu \leq s, 1 \leq j \leq k}}^{\infty} B\left(\mathbf{n}_{1}, \ldots, \mathbf{n}_{s}\right) \prod_{\nu=1}^{s} \prod_{j=1}^{k} \frac{t_{\nu, j}^{n_{\nu, j}}}{n_{\nu, j} !} \\
=\exp \left[2^{-2 \alpha_{s}} \sum_{\substack{\left(\nu_{1}, j_{1}\right) \neq\left(\nu_{2}, j_{2}\right) \\
1 \leq \nu_{1}, \nu_{2} \leq s, 1 \leq j_{1}, j_{2} \leq k}}\left[\sum_{l=1}^{s} \frac{\omega_{\nu_{1}, l} \omega_{\nu_{2}, l}}{a_{l}}\right] t_{\nu_{1}, j_{1}} t_{\nu_{2}, j_{2}}\right] .
\end{gathered}
$$

Proof. By (2.5) and (1.5) the left hand side of (2.8) times $\pi^{s / 2} / \prod_{l=1}^{s} a_{l}^{1 / 2}$ equals

$$
\begin{gathered}
\int_{\mathbb{R}^{s}} e^{-\sum_{l=1}^{s} a_{l} x_{l}^{2}} \prod_{\nu=1}^{s} \prod_{j=1}^{k} \sum_{n_{\nu, j}=0}^{\infty} H_{n_{\nu, j}}\left(L_{\nu}(\vec{x})\right) \frac{\left[2^{-\alpha_{s}} t_{\nu, j}\right]^{n_{\nu, j}}}{n_{\nu, j} !} d x_{1} \cdots d x_{s} \\
=\int_{\mathbb{R}^{s}} e^{-\sum_{l=1}^{s} a_{l} x_{l}^{2}} \prod_{\nu=1}^{s} \prod_{j=1}^{k} \exp \left[2^{1-\alpha_{s}} L_{\nu}(\vec{x}) t_{\nu, j}-2^{-2 \alpha_{s}} t_{\nu, j}^{2}\right] d x_{1} \cdots d x_{s} \\
=\exp \left[-2^{-2 \alpha_{s}} \sum_{\nu=1}^{s} \sum_{j=1}^{k} t_{\nu, j}^{2}\right] \\
\times \int_{\mathbb{R}^{s}} \exp \left[-\sum_{l=1}^{s} a_{l} x_{l}^{2}+2^{1-\alpha_{s}} \sum_{l=1}^{s}\left[\sum_{\nu=1}^{s} \sum_{j=1}^{k} \omega_{\nu, l} t_{\nu, j}\right] x_{l}\right] d x_{1} \cdots d x_{s} \\
=\exp \left[-2^{-2 \alpha_{s}} \sum_{\nu=1}^{s} \sum_{j=1}^{k} t_{\nu, j}^{2}+2^{-2 \alpha_{s}} \sum_{l=1}^{s} \frac{1}{a_{l}}\left[\sum_{\nu=1}^{s} \sum_{j=1}^{k} \omega_{\nu, l} t_{\nu, j}\right]^{2}\right] \\
\quad \times \int_{\mathbb{R}^{s}} \exp \left[-\sum_{l=1}^{s} a_{l}\left[x_{l}-\frac{2^{-\alpha_{s}}}{a_{l}} \sum_{\nu=1}^{s} \sum_{j=1}^{k} \omega_{\nu, l} t_{\nu, j}\right]^{2}\right] d x_{1} \cdots d x_{s} .
\end{gathered}
$$


By (2.7) the exponential expression in line five of (2.9) reduces to the right hand side of (2.8), and by (2.3) the integral in line six of (2.9) reduces to $\prod_{l=1}^{s} \sqrt{\pi / a_{l}}$. This completes the verification of (2.8).

We can now state and prove the following generalization of the two-color multiset combinatorics.

Theorem 2.4. Suppose that we have $s$ multisets colored in $s$ different colors and having $k$ components each. Let $\mathbf{n}_{\nu}=\left(n_{\nu, 1}, \ldots, n_{\nu, k}\right) \in \mathbb{N}_{0}^{k}$ be the size of the color $\nu$ multiset, $\nu=1, \ldots, s$. We match elements from different sets and assign a weight to each pair of matched elements in the following way: When an element from component $j_{1}$ of the color $\nu_{1}$ multiset is matched with an element from component $j_{2}$ of the color $\nu_{2}$ multiset (with $\left(\nu_{1}, j_{1}\right) \neq\left(\nu_{2}, j_{2}\right)$ ), the weight assigned is

$$
2^{1-2 \alpha_{s}} \sum_{l=1}^{s} \frac{\omega_{\nu_{1}, l} \omega_{\nu_{2}, l}}{a_{l}}
$$

where $a_{l}>0, l=1, \ldots, s$ and $\left\{\omega_{\nu, l}, 1 \leq \nu \leq s, 1 \leq l \leq s\right\} \subset \mathbb{C}$ satisfy conditions (2.7), and $\alpha_{s} \geq 0$.

Then the total weight of all such weighted perfect matchings is $B\left(\mathbf{n}_{1}, \ldots, \mathbf{n}_{s}\right)$.

Proof. This result follows from the generating function (2.8) and Theorem 1.4.

The case $s=2$ and $k=1$ is worth recording separately. In this case from (2.5), (2.7), and (2.8) with $\alpha_{s}=\alpha_{2}=1 / 2$ we obtain the orthogonality relation

$$
\int_{\mathbb{R}^{2}} H_{m}(\alpha x+\beta y) H_{n}(\gamma x+\delta y) e^{-a x^{2}-b y^{2}} d x d y=\frac{\pi}{\sqrt{a b}} 2^{n} n !\left(\frac{\alpha \gamma}{a}+\frac{\beta \delta}{b}\right)^{n} \delta_{m, n}
$$

where

$$
a>0, \quad b>0, \quad \frac{\alpha^{2}}{a}+\frac{\beta^{2}}{b}=\frac{\gamma^{2}}{a}+\frac{\delta^{2}}{b}=1 .
$$

The orthogonality relations (1.6) and (1.11) are special cases of (2.11).

2.2. Combinatorics involving colored sets. As an application of Theorem 2.4, we consider the following situation. Suppose that we have a collection of $s$ sets of different colors and sizes $n_{1}, \ldots, n_{s}$. We match elements from different sets and assign weight to each pair of matched elements. When an element from set $\nu_{1}$ is matched with an element from set $\nu_{2}$, the weight assigned is $w_{\nu_{1}, \nu_{2}}, 1 \leq \nu_{1} \neq \nu_{2} \leq s$. The weight of such perfect matching is again the product of the weights of its edges.

Theorem 2.5. Assume that the matrix $W=\left[\delta_{j, l}+\left(1-\delta_{j, l}\right) w_{j, l}\right]_{j, l=1}^{s}$ is real, symmetric, and positive-definite. Then the total weight of all weighted perfect matchings of $s$ colored sets is the number

$$
B(\mathbf{n}, W)=\frac{2^{-\frac{1}{2}|\mathbf{n}|}}{\pi^{s / 2}} \int_{\mathbb{R}^{s}} \prod_{\nu=1}^{s} H_{n_{\nu}}\left(\sum_{l=1}^{s} \omega_{\nu, l} x_{l}\right) e^{-\sum_{l=1}^{s} x_{l}^{2}} d x_{1} \cdots d x_{s}
$$

where $\mathbf{n}=\left(n_{1}, \ldots, n_{s}\right) \in \mathbb{N}^{s}$ and the matrix $\Omega=\left[\omega_{\nu, l}\right]_{\nu, l=1}^{s}$ satisfies the matrix equation

$$
\Omega \Omega^{T}=W
$$


Proof. Since $W$ is positive-definite, equation (2.14) has a solution of the form $\Omega=$ $P^{T} D^{1 / 2}$, where $D$ is a diagonal matrix whose diagonal entries are the eigenvalues of $W, P$ is a unitary matrix whose rows are the corresponding eigenvectors of $W$, and $W=P^{T} D P$. Then Theorem 2.5 follows from Theorems 2.4 and 2.3 with $k=1$, $\alpha_{s}=1 / 2, a_{l}=1, l=1, \ldots, s$, and formulas (2.10) and (2.14).

By Sylvester's criterion, the matrix $W$ is positive-definite if and only if its principal minors are positive. Below we consider the special case when $w_{j, l}=w_{j} w_{l}$, $1 \leq j \neq l \leq s$.

\section{Lemma 2.6.}

$$
\operatorname{det}\left[\delta_{j, l}+\left(1-\delta_{j, l}\right) w_{j} w_{l}\right]_{j, l=1}^{s}=1+\sum_{\nu=2}^{s}(-1)^{\nu-1}(\nu-1) \sigma_{s, \nu}\left(w_{1}^{2}, \ldots, w_{s}^{2}\right)
$$

where $\sigma_{s, \nu}$ denotes the $\nu$-th elementary symmetric function of $s$ variables.

Proof. Let $D_{s}\left(w_{1}, \ldots, w_{s}\right)$ denote the determinant in (2.15). We factor out $w_{j}$ from row $j, j=1, \ldots, s$. Then we multiply column $l$ by $w_{l}, l=1, \ldots, s$. We get

$$
D_{s}\left(w_{1}, \ldots, w_{s}\right)=\operatorname{det}\left[\delta_{j, l}+\left(1-\delta_{j, l}\right) w_{l}^{2}\right]_{j, l=1}^{s} .
$$

Therefore $D_{s}\left(w_{1}, \ldots, w_{s}\right)$ has a unique representation of the form

$$
D_{s}\left(w_{1}, \ldots, w_{s}\right)=1+\sum_{\nu=2}^{s} c_{s, \nu} \sigma_{s, \nu}\left(w_{1}^{2}, \ldots, w_{s}^{2}\right) .
$$

By (2.15) we have $D_{s}\left(w_{1}, \ldots, w_{s-1}, 0\right)=D_{s-1}\left(w_{1}, \ldots, w_{s-1}\right)$, that is,

$$
1+\sum_{\nu=2}^{s-1} c_{s, \nu} \sigma_{s-1, \nu}\left(w_{1}^{2}, \ldots, w_{s-1}^{2}\right)=1+\sum_{\nu=2}^{s-1} c_{s-1, \nu} \sigma_{s-1, \nu}\left(w_{1}^{2}, \ldots, w_{s-1}^{2}\right) .
$$

Hence $c_{s, \nu}=c_{s-1, \nu}, \nu=2, \ldots, s-1$. This implies $c_{s, \nu}=c_{\nu, \nu}, s \geq \nu \geq 2$. So it suffices to show that $c_{\nu, \nu}=(-1)^{\nu-1}(\nu-1)$. Notice that $c_{s, s}$ is the leading coefficient of the polynomial

$$
P_{s}(t)=D_{s}(\sqrt{t}, \ldots, \sqrt{t})=\operatorname{det}\left[\delta_{j, l}+\left(1-\delta_{j, l}\right) t\right]_{j, l=1}^{s} .
$$

It is clear from (2.16) that $P_{s}^{(r)}(1)=0, r=0, \ldots, s-2$. Adding the top $s-1$ rows to the last row in the determinant in (2.16) shows that $(1+(s-1) t)$ divides $P_{s}(t)$. Since the constant term of $P_{s}(t)$ is 1 , we get

$$
P_{s}(t)=(1-t)^{s-1}(1+(s-1) t),
$$

hence $c_{s, s}=(-1)^{s-1}(s-1)$.

Corollary 2.7. A sufficient condition for the positive definiteness of the determinant in (2.15) is $\left|w_{j}\right|<\left((s-2) 2^{s-1}+1\right)^{-1 / 2}, j=1, \ldots, s$. 
3. The Combinatorics of $\left\{H_{m, n}(z, \bar{z})\right\}$

Let $\mathbf{m}_{\nu}=\left(m_{\nu, 1}, \ldots, m_{\nu, k}\right) \in \mathbb{N}_{0}^{k}, \mathbf{n}_{\nu}=\left(n_{\nu, 1}, \ldots, n_{\nu, k}\right) \in \mathbb{N}_{0}^{k}, \nu=1, \ldots, s$. Consider the integrals

$$
\begin{aligned}
I\left(\mathbf{m}_{1}, \mathbf{n}_{1}, \ldots, \mathbf{m}_{s}, \mathbf{n}_{s}\right) & =\pi^{-s / 2} \int_{\mathbb{R}^{s}} \prod_{\nu=1}^{s} \prod_{j=1}^{k} H_{m_{\nu, j}, n_{\nu, j}}\left(L_{\nu}(\vec{x}), \overline{L_{\nu}(\vec{x})}\right) \\
& \times e^{-\sum_{l=1}^{s} x_{l}^{2}} d x_{1} \cdots d x_{s},
\end{aligned}
$$

where $\vec{x}=\left(x_{1}, \ldots, x_{s}\right)$ and $L_{\nu}(\vec{x})=\sum_{l=1}^{s} \omega_{\nu, l} x_{l}, \nu=1, \ldots, s$, with complex coefficients satisfying

$$
\sum_{l=1}^{s} \omega_{\nu, l}^{2}=0, \quad \nu=1, \ldots, s .
$$

For vectors $\vec{\omega}=\left(\omega_{1}, \ldots, \omega_{s}\right) \in \mathbb{C}^{s}$ and $\vec{\eta}=\left(\eta_{1}, \ldots, \eta_{s}\right) \in \mathbb{C}^{s}$ we set $\vec{\omega} \vec{\eta}=\sum_{l=1}^{s} \omega_{l} \eta_{l}$.

\section{Theorem 3.1.}

$$
\begin{aligned}
& \sum_{\substack{m_{\nu, j}, n_{\nu, j}=0 \\
1 \leq \nu \leq s, 1 \leq j \leq k}}^{\infty} I\left(\mathbf{m}_{1}, \mathbf{n}_{1}, \ldots, \mathbf{m}_{s}, \mathbf{n}_{s}\right) \prod_{\nu=1}^{s} \prod_{j=1}^{k} \frac{t_{\nu, j}^{m_{\nu, j}} w_{\nu, j}^{n_{\nu, j}}}{m_{\nu, j} ! n_{\nu, j} !} \\
& =\exp \left[\frac{1}{4} \sum_{\substack{\left(\nu_{1}, j_{1}\right) \neq\left(\nu_{2}, j_{2}\right) \\
1 \leq \nu_{1}, \nu_{2} \leq s \\
1 \leq j_{1}, j_{2} \leq k}}\left[\vec{\omega}_{\nu_{1}} \vec{\omega}_{\nu_{2}} t_{\nu_{1}, j_{1}} t_{\nu_{2}, j_{2}}+\overline{\vec{\omega}_{\nu_{1}}} \overline{\vec{\omega}_{\nu_{2}}} w_{\nu_{1}, j_{1}} w_{\nu_{2}, j_{2}}+2 \vec{\omega}_{\nu_{1}} \overline{\vec{\omega}_{\nu_{2}}} t_{\nu_{1}, j_{1}} w_{\nu_{2}, j_{2}}\right]\right] \\
& \times \exp \left[\sum_{\nu=1}^{s} \sum_{j=1}^{k}\left(\frac{1}{2} \vec{\omega}_{\nu} \overline{\vec{\omega}}_{\nu}-1\right) t_{\nu, j} w_{\nu, j}\right] .
\end{aligned}
$$

Proof. By (3.1) and (1.2) the left hand side of (3.3) times $\pi^{s / 2}$ equals

$$
\begin{gathered}
\int_{\mathbb{R}^{s}} \prod_{\nu=1}^{s} \prod_{j=1}^{k}\left[\sum_{m_{\nu, j}=0}^{\infty} \sum_{n_{\nu, j}=0}^{\infty} H_{m_{\nu, j} n_{\nu, j}}\left(L_{\nu}(\vec{x}), \overline{L_{\nu}(\vec{x})}\right) \frac{t_{\nu, j}^{m_{\nu, j}} w_{\nu, j}^{n_{\nu, j}}}{m_{\nu, j} ! n_{\nu, j} !}\right] e^{-\sum_{l=1}^{s} x_{l}^{2}} d x_{1} \cdots d x_{s} \\
=\int_{\mathbb{R}^{s}} \exp \left[\sum_{\nu=1}^{s}\left[L_{\nu}(\vec{x}) \sum_{j=1}^{k} t_{\nu, j}+\overline{L_{\nu}(\vec{x})} \sum_{j=1}^{k} w_{\nu, j}-\sum_{j=1}^{k} t_{\nu, j} w_{\nu, j}\right]-\sum_{l=1}^{s} x_{l}^{2}\right] d x_{1} \cdots d x_{s} \\
=\int_{\mathbb{R}^{s}} \exp \left[\sum_{l=1}^{s}\left[-x_{l}^{2}+\sum_{\nu=1}^{s} \sum_{j=1}^{k}\left(\omega_{\nu, l} t_{\nu, j}+\overline{\omega_{\nu, l}} w_{\nu, j}\right) x_{l}\right]-\sum_{\nu=1}^{s} \sum_{j=1}^{k} t_{\nu, j} w_{\nu, j}\right] d x_{1} \cdots d x_{s} \\
=\exp \left[\frac{1}{4} \sum_{l=1}^{s}\left[\sum_{\nu=1}^{s} \sum_{j=1}^{k}\left(\omega_{\nu, l} t_{\nu, j}+\overline{\omega_{\nu, l}} w_{\nu, j}\right)\right]^{2}-\sum_{\nu=1}^{s} \sum_{j=1}^{k} t_{\nu, j} w_{\nu, j}\right] \\
\times \prod_{l=1}^{s} \int_{\mathbb{R}} \exp \left[-\left[x_{l}-\frac{1}{2} \sum_{\nu=1}^{s} \sum_{j=1}^{k}\left(\omega_{\nu, l} t_{\nu, j}+\overline{\omega_{\nu, l}} w_{\nu, j}\right)\right] d x_{l} .\right.
\end{gathered}
$$

By (3.2) the exponential expression in line four of (3.4) reduces to the exponential expression on the right hand side of (3.3), while by (2.3) each integral in line five of (3.4) equals $\sqrt{\pi}$. 
The combinatorial interpretation of the polynomials $\left\{H_{m, n}(z, \bar{z})\right\}$ follows.

Theorem 3.2. Suppose that we have $s$ color I multisets $B_{1}, \ldots, B_{s}$ and $s$ color II multisets $R_{1}, \ldots, R_{s}$, each having $k$ components. Multisets $B_{1}, \ldots, B_{s}$ have sizes $\mathbf{m}_{1}, \ldots, \mathbf{m}_{s} \in \mathbb{N}_{0}^{k}$ and multisets $R_{1}, \ldots, R_{s}$ have sizes $\mathbf{n}_{1}, \ldots, \mathbf{n}_{s} \in \mathbb{N}_{0}^{k}$, respectively. Consider all inhomogeneous perfect matchings of these $2 s$ multisets with weights assigned in the following way: If the matched elements are from components $j_{1}$ of $B_{\nu_{1}}$ and $j_{2}$ of $B_{\nu_{2}}$, the weight is $\frac{1}{2} \vec{\omega}_{\nu_{1}} \vec{\omega}_{\nu_{2}}$; if they are from components $j_{1}$ of $R_{\nu_{1}}$ and $j_{2}$ of $R_{\nu_{2}}$, the weight is $\frac{1}{2} \overline{\vec{\omega}}_{\nu_{1}} \overrightarrow{\vec{\omega}}_{\nu_{2}}$; if they are from components $j_{1}$ of $B_{\nu_{1}}$ and $j_{2}$ of $R_{\nu_{2}}$, the weight is $\frac{1}{2} \vec{\omega}_{\nu_{1}} \frac{\vec{\omega}_{\nu_{2}}}{\omega_{1}}-\delta_{\nu_{1}, \nu_{2}} \delta_{j_{1}, j_{2}}$.

The total weight of all such weighted perfect matchings is $I\left(\mathbf{m}_{1}, \mathbf{n}_{1}, \ldots, \mathbf{m}_{s}, \mathbf{n}_{s}\right)$.

Proof. The theorem follows from the generating function (3.3) and Theorem 1.4.

Corollary 3.3. Let $\mathbf{m}, \mathbf{n}, \mathbf{p}, \mathbf{q} \in \mathbb{N}_{0}^{k}$. The integrals in (1.12) have the exponential generating function

$$
\begin{aligned}
& \sum_{\substack{m_{j}, n_{j}, p_{j}, q_{j}=0 \\
1 \leq j \leq k}}^{\infty} I(\mathbf{m}, \mathbf{n}, \mathbf{p}, \mathbf{q}) \prod_{j=1}^{k} \frac{u_{j}^{m_{j}} v_{j}^{n_{j}} w_{j}^{p_{j}} t_{j}^{q_{j}}}{m_{j} ! n_{j} ! p_{j} ! q_{j} !} \\
& =\exp \left[\sum_{1 \leq j_{1} \neq j_{2} \leq k}\left(u_{j_{1}}+w_{j_{1}}\right)\left(v_{j_{2}}+t_{j_{2}}\right)+\sum_{j=1}^{k}\left(u_{j} t_{j}+v_{j} w_{j}\right)\right] .
\end{aligned}
$$

This result follows immediately from Theorem 3.1 with $s=2, \mathbf{m}_{1}=\mathbf{m}, \mathbf{n}_{1}=\mathbf{n}$, $\mathbf{m}_{2}=\mathbf{p}, \mathbf{n}_{2}=\mathbf{q}$, variables $t_{1, j}=u_{j}, w_{1, j}=v_{j}, t_{2, j}=w_{j}, w_{2, j}=t_{j}, j=1, \ldots, k$, $x_{1}=x, x_{2}=y$, and linear functions $L_{1}(\vec{x})=L_{2}(\vec{x})=x+i y$.

Proof of Theorem 1.3. This theorem is a special case of Theorem 3.2 with $s=k=2$ and $\vec{\omega}_{1}=\vec{\omega}_{2}=(1, i)$. It also follows from the generating function (3.5) and Theorem 1.4 .

Corollary 3.4. The polynomials $\left\{H_{m, n}(z, \bar{z})\right\}$ satisfy the orthogonality relation

$$
\frac{1}{\pi} \int_{\mathbb{R}^{2}} H_{m, n}(x+i y, x-i y) H_{p, q}(x+i y, x-i y) e^{-x^{2}-y^{2}} d x d y=m ! n ! \delta_{m, q} \delta_{n, p} .
$$

This corollary is an immediate consequence of the case $k=1$ of equations (1.12) and (3.5).

It is important to note that (1.3) implies the symmetry relation

$$
\overline{H_{m, n}(z, \bar{z})}=H_{n, m}(z, \bar{z}) \text {. }
$$

Then orthogonality relation (1.8) follows from equations (3.6) and (3.7).

\section{INTEGRAL OPERATORS}

In this section we discuss integral operators related to the Poisson kernel for $\left\{H_{n}(z)\right\}$. Integral operators associated with the polynomials $\left\{H_{m, n}(z, \bar{z})\right\}$ are studied in 12 .

The Poisson kernel for the polynomials $\left\{H_{n}(z)\right\}$ is ([11, Section 4.7]),

$$
K_{r}(z, w)=\sum_{n=0}^{\infty} H_{n}(z) H_{n}(w) \frac{r^{n}}{2^{n} n !}=\left(1-r^{2}\right)^{-1 / 2} \exp \left(\frac{2 z w r-\left(z^{2}+w^{2}\right) r^{2}}{1-r^{2}}\right) .
$$


Let $a$ and $b$ satisfy conditions (1.7). Define the integral operator $T_{1}$ by

$$
\left(T_{1} f\right)(x+i y)=\frac{\sqrt{a b}}{\pi} \int_{\mathbb{R}^{2}} K_{r}(x+i y, \xi-i \eta) f(\xi+i \eta) e^{-a \xi^{2}-b \eta^{2}} d \xi d \eta
$$

It is clear from (4.1) and (1.6) that

$$
\left(T_{1} H_{n}\right)(z)=\left(\frac{a+b}{a b}\right)^{n} r^{n} H_{n}(z), \quad n \in \mathbb{N}_{0} .
$$

Another natural integral operator to consider is

$$
\left(T_{2} f\right)(x+i y)=\frac{\sqrt{a b}}{\pi} \int_{\mathbb{R}^{2}} K_{r}(x+i y, \xi-i \eta) f(\xi-i \eta) e^{-a \xi^{2}-b \eta^{2}} d \xi d \eta
$$

Similarly, from (4.1) and (1.11) it follows that

$$
\left(T_{2} H_{n}\right)(z)=r^{n} H_{n}(z), \quad n \in \mathbb{N}_{0} .
$$

In order to justify the analysis leading to (4.3) and (4.5), we assume $|r|<1$. On the other hand it is known ([11, Section 4.6]) that the Fourier transform has eigenvalues $i^{n}$ and eigenfunctions $e^{-x^{2} / 2} H_{n}(x)$. Thus one is tempted to let $r=i$ in (4.2) and (4.4). This is indeed possible and we state and prove this fact in Theorem 4.1 below. The proof of Theorem 4.1 uses the representation of the monomials in terms of the Hermite polynomials. Multiplying both sides of equation (1.5) by $e^{t^{2}}$ and equating the coefficients of $t^{n}$ on both sides of the resulting equation, we obtain

$$
z^{n}=\frac{n !}{2^{n}} \sum_{k=0}^{\lfloor n / 2\rfloor} \frac{H_{n-2 k}(z)}{k !(n-2 k) !}, \quad n \in \mathbb{N}_{0}
$$

\section{Theorem 4.1.}

$$
\frac{\sqrt{a b}}{\pi \sqrt{2}} \int_{\mathbb{R}^{2}} e^{i z(\xi-i \eta)+\left(z^{2}+(\xi-i \eta)^{2}\right) / 2} H_{n}(\xi+i \eta) e^{-a \xi^{2}-b \eta^{2}} d \xi d \eta=\left(\frac{a+b}{a b} i\right)^{n} H_{n}(z),
$$

$$
\frac{\sqrt{a b}}{\pi \sqrt{2}} \int_{\mathbb{R}^{2}} e^{i z(\xi-i \eta)+\left(z^{2}+(\xi-i \eta)^{2}\right) / 2} H_{n}(\xi-i \eta) e^{-a \xi^{2}-b \eta^{2}} d \xi d \eta=i^{n} H_{n}(z)
$$

$n \in \mathbb{N}_{0}$, where $a$ and $b$ satisfy conditions (1.7). 
Proof. The left hand sides of (4.7)-(4.8) are

$$
\begin{gathered}
\frac{\sqrt{a b}}{\pi \sqrt{2}} e^{z^{2} / 2} \int_{\mathbb{R}^{2}} \sum_{m=0}^{\infty} \frac{H_{m}(z / \sqrt{2})}{m !}\left(\frac{i(\xi-i \eta)}{\sqrt{2}}\right)^{m} H_{n}(\xi \pm i \eta) e^{-a \xi^{2}-b \eta^{2}} d \xi d \eta \\
=\frac{\sqrt{a b}}{\pi \sqrt{2}} e^{z^{2} / 2} \sum_{m=0}^{\infty} \frac{H_{m}(z / \sqrt{2})}{m !}\left(\frac{i}{\sqrt{2}}\right)^{m} \\
\quad \times \int_{\mathbb{R}^{2}}\left(\frac{m !}{2^{m}} \sum_{k=0}^{\lfloor m / 2\rfloor} \frac{H_{m-2 k}(\xi-i \eta)}{k !(m-2 k) !}\right) H_{n}(\xi \pm i \eta) e^{-a \xi^{2}-b \eta^{2}} d \xi d \eta \\
=\frac{\sqrt{a b}}{\pi \sqrt{2}} e^{z^{2} / 2} \sum_{j=0}^{\infty} H_{n+2 j}(z / \sqrt{2})\left(\frac{i}{2 \sqrt{2}}\right)^{n+2 j} \frac{1}{j ! n !} \frac{\pi}{\sqrt{a b}} 2^{n} n !\left(\frac{b \pm a}{a b}\right)^{n} \\
=\frac{1}{\sqrt{2}}\left(\frac{b \pm a}{a b \sqrt{2}} i\right)^{n} e^{z^{2} / 2} \sum_{j=0}^{\infty} \frac{H_{n+2 j}(z / \sqrt{2})}{j !}(-1 / 8)^{j}
\end{gathered}
$$

where we applied (1.5) to get the first line, (4.6) to get the third line, and the orthogonality relations (1.6) and (1.11) to get the fourth line. To evaluate the $j$-sum in the last line of (4.9) we use the integral representation ([11, Equation $(4.6 .41)])$,

$$
H_{n}(i z)=\frac{(2 i)^{n}}{\sqrt{\pi}} \int_{\mathbb{R}} e^{-(u-z)^{2}} u^{n} d u .
$$

Formula (4.10) leads to the evaluation of the $j$-sum in the following way:

$$
\begin{gathered}
\sum_{j=0}^{\infty} \frac{H_{n+2 j}(z / \sqrt{2})}{j !}(-1 / 8)^{j}=\frac{1}{\sqrt{\pi}} \int_{\mathbb{R}} e^{-(u+i z / \sqrt{2})^{2}} \sum_{j=0}^{\infty} \frac{(2 i u)^{n+2 j}(-1 / 8)^{j}}{j !} d u \\
=\frac{(2 i)^{n}}{\sqrt{\pi}} \int_{\mathbb{R}} e^{-(u+i z / \sqrt{2})^{2}+u^{2} / 2} u^{n} d u=\frac{(2 i)^{n}}{\sqrt{\pi}} e^{-z^{2} / 2} \int_{\mathbb{R}} e^{-(u / \sqrt{2}+i z)^{2}} u^{n} d u \\
=\frac{(2 i)^{n}}{\sqrt{\pi}} e^{-z^{2} / 2}(\sqrt{2})^{n+1} \int_{\mathbb{R}} e^{-(v+i z)^{2}} v^{n} d v=(\sqrt{2})^{n+1} e^{-z^{2} / 2} H_{n}(z) .
\end{gathered}
$$

Formulas (4.7)-(4.8) now follow from (4.9) and (4.11).

Corollary 4.2. Consider the integral operators

$$
\left(F_{1,2} f\right)(z)=\frac{\sqrt{a b}}{\pi \sqrt{2}} \int_{\mathbb{R}^{2}} e^{i z(\xi-i \eta)+\left(z^{2}+(\xi-i \eta)^{2}\right) / 2} f(\xi \pm i \eta) e^{-a \xi^{2}-b \eta^{2}} d \xi d \eta
$$

where $a>1 / 2$ and $b>1 / 2$ satisfy conditions (1.7). Then for every $n \in \mathbb{N}_{0}$ the polynomial $H_{n}(z)$ is an eigenfunction of the operators $F_{1}$ and $F_{2}$ with eigenvalues $\left(\frac{a+b}{a b} i\right)^{n}$ and $i^{n}$, respectively.

One important problem is the expansion of functions in suitable bases for Hilbert spaces.

Theorem 4.3. The polynomials $\left\{H_{m}(x+i y) H_{n}(x-i y), m, n \in \mathbb{N}_{0}\right\}$ form a basis for the Hilbert space $L_{2}\left(\mathbb{R}^{2}, e^{-a x^{2}-b y^{2}}\right)$ with $a$ and $b$ related via (1.7).

Proof. Clearly the polynomials $\left\{H_{m}(x+i y) H_{n}(x-i y), m, n \in \mathbb{N}_{0}\right\}$ span the space of all polynomials of $x$ and $y$. Let $f \in L_{2}\left(\mathbb{R}^{2}, e^{-a x^{2}-b y^{2}}\right)$. We may assume that $f$ 
has compact support in which case it is easy to see that $f$ can be uniformly approximated on $\mathbb{R}^{2}$ by linear combinations of functions of the form $g(x) h(y)$ where $g$ and $h$ are continuous functions of compact support. But each of these latter functions can be uniformly approximated on $\mathbb{R}$ by weighted polynomials $e^{-a x^{2}} p_{m}(x)$ and $e^{-b y^{2}} q_{n}(y)$. Then, the linear combinations of $g(x) h(y)$ are uniformly approximated on $\mathbb{R}^{2}$ by the corresponding linear combination of the products of these weighted polynomials.

Finally, we record the formulas for the moments of $e^{-a x^{2}-b y^{2}}$ with $a$ and $b$ related via (1.7).

\section{Proposition 4.4 .}

$$
\begin{gathered}
\int_{\mathbb{R}^{2}}(x \pm i y)^{2 n+1} e^{-a x^{2}-b y^{2}} d x d y=0, \\
\int_{\mathbb{R}^{2}}(x \pm i y)^{2 n} e^{-a x^{2}-b y^{2}} d x d y=\frac{\pi}{\sqrt{a b}}(1 / 2)_{n} .
\end{gathered}
$$

Proof. By orthogonality relation (1.11) and the fact that $H_{0}(z)=1$, the moment of $(x \pm y)^{n}$ is the integral of the coefficient of $H_{0}(x \pm i y)$ on the right hand side of (4.6).

\section{ACKNOWLEDGMENTS}

This work started at the XXIX International Colloquium on Group-Theoretic Methods in Physics where Andrzej Horzela spoke about the 2D-Hermite polynomials and raised the question of studying their combinatorics. The authors thank S. Twareque Ali, Andrzej Horzela, Karol Penson, and Franciszek Szafraniec for references and discussions. The authors thank Vilmos Totik for the argument in Theorem 4.3 .

\section{REFERENCES}

[1] S. Twareque Ali, F. Bagarello, and G. Honnouvo, Modular structures on trace class operators and applications to Landau levels, J. Phys. A 43 (2010), no. 10, 105202, 17, DOI 10.1088/1751-8113/43/10/105202. MR2593994(2011h:81115)

[2] Richard Askey, Orthogonal polynomials and special functions, Society for Industrial and Applied Mathematics, Philadelphia, Pa., 1975. MR0481145 (58 \#1288)

[3] Ruth Azor, J. Gillis, and J. D. Victor, Combinatorial applications of Hermite polynomials, SIAM J. Math. Anal. 13 (1982), no. 5, 879-890, DOI 10.1137/0513062. MR668329 (84d:33012)

[4] Nicolae Cotfas, Jean Pierre Gazeau, and Katarzyna Górska, Complex and real Hermite polynomials and related quantizations, J. Phys. A 43 (2010), no. 30, 305304, 14, DOI 10.1088/1751-8113/43/30/305304. MR2659624(2011h:81121)

[5] Charles F. Dunkl and Yuan Xu, Orthogonal polynomials of several variables, Encyclopedia of Mathematics and its Applications, vol. 81, Cambridge University Press, Cambridge, 2001. MR:1827871 (2002m:33001)

[6] Jean Pierre Gazeau and Franciszek Hugon Szafraniec, Holomorphic Hermite polynomials and a non-commutative plane, J. Phys. A 44 (2011), no. 49, 495201, 13, DOI 10.1088/17518113/44/49/495201. MR2860876 (2012m:81099)

[7] Allal Ghanmi, A class of generalized complex Hermite polynomials, J. Math. Anal. Appl. 340 (2008), no. 2, 1395-1406, DOI 10.1016/j.jmaa.2007.10.001. MR2390939 (2009e:33025)

[8] A. Ghanmi, Operational formulae for the complex Hermite polynomials $H_{p, q}(z, \bar{z})$, Integral Transforms and Special Functions 340 (2013).

[9] C. D. Godsil, Hermite polynomials and a duality relation for matching polynomials, Combinatorica 1 (1981), no. 3, 257-262, DOI 10.1007/BF02579331. MR637830 (83e:05015) 
[10] K. Gorska, private communication.

[11] Mourad E. H. Ismail, Classical and quantum orthogonal polynomials in one variable, Encyclopedia of Mathematics and its Applications, vol. 98, Cambridge University Press, Cambridge, 2005. With two chapters by Walter Van Assche; With a foreword by Richard A. Askey. MR2191786 (2007f:33001)

[12] Mourad E. H. Ismail, Analytic properties of complex Hermite polynomials, preprint, 2013.

[13] Mourad E. H. Ismail and Plamen Simeonov, Asymptotics of generalized derangements, Adv. Comput. Math. 39 (2013), no. 1, 101-127, DOI 10.1007/s10444-011-9271-7. MR3068596

[14] Kiyosi Itô, Complex multiple Wiener integral, Jap. J. Math. 22 (1952), 63-86 (1953). MR0063609 (16,151b)

[15] Dmitri Karp, Holomorphic spaces related to orthogonal polynomials and analytic continuation of functions, Analytic extension formulas and their applications (Fukuoka, 1999/Kyoto, 2000), Int. Soc. Anal. Appl. Comput., vol. 9, Kluwer Acad. Publ., Dordrecht, 2001, pp. 169-187. MR:1830382(2002c:46054)

[16] Dongsu Kim and Jiang Zeng, A combinatorial formula for the linearization coefficients of general Sheffer polynomials, European J. Combin. 22 (2001), no. 3, 313-332, DOI 10.1006/eujc.2000.0459. MR1822720 (2001k:05023)

[17] Richard P. Stanley, Enumerative combinatorics. Vol. 2, Cambridge Studies in Advanced Mathematics, vol. 62, Cambridge University Press, Cambridge, 1999. With a foreword by Gian-Carlo Rota and appendix 1 by Sergey Fomin. MR.1676282 (2000k:05026)

[18] Gábor Szegő, Orthogonal polynomials, 4th ed., American Mathematical Society, Providence, R.I., 1975. American Mathematical Society, Colloquium Publications, Vol. XXIII. MR0372517 (51 \#8724)

[19] K. Thirulogasanthar, G. Honnouvo, and A. Krzyżak, Coherent states and Hermite polynomials on quaternionic Hilbert spaces, J. Phys. A 43 (2010), no. 38, 385205, 13, DOI 10.1088/1751-8113/43/38/385205. MR2718325 (2012c:81106)

[20] S. J. L. van Eijndhoven and J. L. H. Meyers, New orthogonality relations for the Hermite polynomials and related Hilbert spaces, J. Math. Anal. Appl. 146 (1990), no. 1, 89-98, DOI 10.1016/0022-247X(90)90334-C. MR1041203 (91i:33004)

[21] X. G. Viennot, Une théorie combinatoire des polynômes orthogonaux généraux, Lecture Notes, Université du Québec à Montréal, Montreal, 1983.

[22] Jiang Zeng, Weighted derangements and the linearization coefficients of orthogonal Sheffer polynomials, Proc. London Math. Soc. (3) 65 (1992), no. 1, 1-22, DOI 10.1112/plms/s365.1.1. MR 1162485 (93c:05003)

Department of Mathematics, University of Central Florida, Orlando, Florida 32816 - ANd - King Saud University, Riyadh, SAudi Arabia

E-mail address: mourad.eh.ismail@gmail.com

Department of Mathematics and Statistics, University of Houston-Downtown, HousTON, TEXAS 77002

E-mail address: simeonovp@uhd.edu 